Case Report

\title{
The Importance of Diagnosis and Treatment of May-Thurner and Pelvic Congestion Syndromes Before Complications, in Patients with Thrombophilia
}

\author{
Diego Victor Nascimento ${ }^{1}$, Gabrielle Maria de Oliveira Kraychete da Silveira ${ }^{1}$, \\ Helena Chaves de Queiroga ${ }^{1}$, Lidie Anne Diniz Viégas ${ }^{1}$, Sérgio Ricardo Ferreira Vieira ${ }^{2}$, \\ Francisco Chavier Vieira Bandeira ${ }^{3}$, Paulo Roberto da Silva Lima ${ }^{1, *}$ \\ ${ }^{1}$ Department of Medicine, Universetary Center of João Pessoa (UNIPÊ), João Pessoa - PB, Brazil \\ ${ }^{2}$ Department of Medicine, Faculty of Medical Sciences of Paraíba (FCM), João Pessoa - PB, Brazil \\ ${ }^{3}$ Department of Medicine, Federal University of Paraíba (UFPB), João Pessoa - PB, Brazil
}

Email address:

paulovascular@hotmail.com (P. R. da S. Lima)

${ }^{*}$ Corresponding author

\section{To cite this article:}

Diego Victor Nascimento, Gabrielle Maria de Oliveira Kraychete da Silveira, Helena Chaves de Queiroga, Lidie Anne Diniz Viégas, Sérgio Ricardo Ferreira Vieira, Francisco Chavier Vieira Bandeira, Paulo Roberto da Silva Lima. The Importance of Diagnosis and Treatment of May-Thurner and Pelvic Congestion Syndromes Before Complications, in Patients with Thrombophilia. Journal of Surgery.

Vol. 6, No. 4, 2018, pp. 88-91. doi: 10.11648/j.js.20180604.12

Received: May 1, 2018; Accepted: May 28, 2018; Published: June 14, 2018

\begin{abstract}
May-Thurner syndrome (MTS), initially observed in 1851 by Virchow as an anatomical variation of the left common iliac vein. In 1965, Cockett and Thomas supplemented the work of May and Thurner correlating with the observed symptoms. The syndrome is characterized by an anatomical anomaly that results in compression of the left common iliac vein (LCIV) by the right common iliac artery (RCIA) [3]. It is also known as Cockett Syndrome, iliac vein compression syndrome and iliocaval compression syndrome [3]. Because of the stress arising from the pulsation of the artery, the intima layer of the vein undergoes a hypertrophy with consequent development of Deep Vein Thrombosis (DVT) [1,3]. Because it prevents drainage to the inferior vena cava, MTS is related to the appearance of pelvic varices characterized by dilatation and reflux, venous stasis of the pelvic organs, having as main manifestation chronic pelvic pain (CPP) without inflammatory signs, although the CPP does not is a well-defined diagnostic criterion [5,6]. In substitution for open surgery, appeared more effective methods such as endovascular surgery [7]. The patient in this case authorized the authors to describe her clinical case. We performed a search on the PubMed and Bireme databases. A white woman was complaining of pain in her lower extremities (LE), apart from feeling of heaviness and tiredness in her Right Lower Extremity (RLE), ankle edema, legs and pelvic varices. At physical examination, the patient had the presence (according to the CEAP classification) of C1, 3 varicose veins in the RLE and C1, 2, 3 varicose veins in the Left Lower Extremity (LLE). She was submitted to varied clinical treatment, and the investigation of the causes of the symptoms, including thrombophilia. She was not responding well to the treatment and the endovascular treatment of Cockett's syndrome was performed. Due to the non-release of the embolization of the ovarian veins by the patient's health plan there was a delaying for the right treatment of the patient, because she has ovarian varicose veins. The endovascular treatment of the May-Thurner and Pelvic Congestion Syndrome is safe and has excellent primary patency in the medium to long term.
\end{abstract}

Keywords: May-Thurner Syndrome, Venous Insufficiency, Pelvic, Cockett Symdrome, Therapeutic, Trombophilia

\section{Introduction}

May-Thurner syndrome (MTS), initially observed in 1851 by Virchow as an anatomical variation of the left common iliac vein, had its pathophysiology described by May and Thurner in 1956, which also detailed anatomical description 
of this compression. In 1965, Cockett and Thomas supplemented the work of May and Thurner correlating with the observed symptoms, evidencing how a small change can cause such an important injury [1-3]. The syndrome is characterized by an anatomical anomaly that results in compression of the left common iliac vein (LCIV) by the right common iliac artery (RCIA), although cases of right vein compression have already been reported [3]. It is also known as Cockett Syndrome, iliac vein compression syndrome and iliocaval compression syndrome [3]. LCIV is partially compressed between the RCIA and the fifth lumbar vertebra, and because of the stress arising from the pulsation of the artery, the intima layer of the vein undergoes a hypertrophy with consequent development of Deep Vein Thrombosis (DVT) $[1,3]$. The overall prevalence of MTS varies between 18 and $49 \%$ in patients with lower left DVT and in up to $25 \%$ of healthy individuals, more than $50 \%$ of compression can be observed [2]. Most prevalent in women aged 20-50 [4]. Physical examination reveals, eventually, skin changes that indicate chronic venous insufficiency such as varicose veins, lipodermatosclerosis, phlebopathic ulcers, and moderate edema. Risk factors such as prolonged immobility, recent surgeries and pregnancy are predisposing to the development of MTS. Because it prevents drainage to the inferior vena cava, MTS is related to the appearance of pelvic varices characterized by dilatation and reflux, venous stasis of the pelvic organs, having as main manifestation chronic pelvic pain (CPP) without inflammatory signs, although the CPP does not is a well-defined diagnostic criterion [5, 6]. The available treatments for MTS are the conservative one that consists in the control of the pain, depending or not of a prophylaxis directed towards DVT and the surgical one that provides a decompression of the common left iliac vein. In substitution for open surgery, more effective methods such as endovascular surgery with uncoated stent implantation, in addition to iliac vein adhesiolysis, anastomosis after RCIA transection of LCIV, resection of the involved segment of the iliac vein and vascular graft in situ have been used [7].

\section{Materials and Methods}

The patient in this case authorized the authors to describe her clinical case. We performed a search on the PubMed and Bireme databases, where when we use the keyword (may thurner syndrome treatment) we have 215 articles, if we use the search (pelvic congestion) we have 474 articles, but if we use the search (thrombophilia) AND (may thurner syndrome treatment) we have only 5 articles. This work is a case report with ((pelvic congestion) AND thrombophilia) AND (may thurner syndrome treatment).

\section{Results}

On 11/2007, a 49-year-old white woman was complaining of pain in her lower extremities (LE), apart from feeling of heaviness and tiredness in her Right Lower Extremity (RLE), ankle edema, legs and pelvic varices. The patient had two previous pregnancies and sclerotherapy in varicose vein of her legs. At physical examination, the patient was in good general condition, eupneic, afebrile, lucid, active, oriented, normocorated, normohydrated, without alterations in the her respiratory, cardiac and gastrointestinal systems. In her vascular evaluation noted of absence of arteriopathies and presence (according to the CEAP classification) of $\mathrm{C} 1,3$ varicose veins in the RLE and C1, 2, 3 varicose veins in the Left Lower Extremity (LLE). The performance of vein sclerotherapy, use of compression stockings $3 / 4$ of medium and diosmin with hesperidin were oriented to her. Venous ultrasonography of the lower limbs was requested that revealed only superficial varicose veins and absence of perforating and insufficient ones, being prescripted Mucopolysaccharide Polysulphate Gel $500 \mathrm{mg} 4$ times a day on her legs. In the evolution of the case, the patient reported legs edema in the evening, which disappeared at dawn; her RLE pain was in the posterior region of the thigh. In addition, she had an Abdominal Ultrasonography demonstrating pelvic varices. It was prescribed diosmin + hesperindine $500 \mathrm{mg}$ twice a day and surgery of varicose veins in LLE. Progressed with worsening varicose veins, her varicose veins increased to medium caliber on both legs and appearing of hemorrhoids. In $12 / 2008$, due to the suspicion of MTS and / or pelvic congestion, angioresonance of the abdominal and pelvic vessels was requested (Figure 1), which confirmed this diagnostic hypothesis and ratified its correction. Angioplasty with uncoated stent was requested for treatment of Cockett Syndrome and embolization of varicose ovarian veins, but the health insurance did not authorize the embolization of varicose ovarian veins, only the angioplasty with uncoated stent for treat the compression over the Left Common Iliac Vein. Three months after surgery (05/2009 - Angioplasty), the patient reported that even with the compression stockings, she was still presented a sensation of weight in the legs, but now without edema. It was recommended to her the maintenance of socks and a medicine made of Coumarin + Troxerrutin in case of sensation of weight and fatigue in her legs. After one year, she brought ultrasound (US) of iliac veins that consisted of patent stent and absence of abnormalities in treated vessels by angioplasty (Figure 2). The investigation of vasculitis and scleroderma were negative, but thrombophilia was confirmed by mutation $\mathrm{A} 1298 \mathrm{C}$ and $\mathrm{C} 677 \mathrm{~T}$. She has come back to complained of edema, pain, fatigue, weight sensation in the lower extremities, more pronounced on the left, and could no longer tolerate the use of compressive stockings. At physical examination: varicose veins C1, 2, 3 in LE. A venous Doppler US of her legs was ordered and which revealed bilateral intramuscular deep venous stasis predominantly in the left soleus veins. Due to the persistence of pain and sensation of pulsation in the legs, a new venous angiotomography of the abdomen and pelvis was requested. The result (04/04/2014) revealed that the left ovarian vein was well dilated with a caliber of the aorta and varicose veins on the uterine fund, proceeding with an indication of embolization of the pelvic varicose veins (Figure 3), only performed after judicial release. After embolization of the ovarian veins, the patient still had 
complaints of tiredness in the lower extremities, but without edema. One year later, the initial complaints were terminated and she currently has $\mathrm{C} 1$ varicose veins (Classification CEAP) in both legs and a not well defined sensation in the lower abdomen, in follow-up with gynecology. She also has some reddish spots (telangiectatic clouds). She is now (08/2017) in use of only with acetylsalicylic acid $100 \mathrm{mg}$ /day and wearing socks 7/8 20-30 mmHg.

\section{Discussion}

The MTS treatment of the patient in question was based on angioplasty with uncoated stent and posterior embolization of the ovarian veins; and in the use of full anticoagulation for 2 years with subsequent maintenance with antiplatelet, to avoid stent occlusion due to hyperplasia and / or thrombosis. The most common form of clinical presentation is acute ileofemoral DVT. It may also have the form of progressive edema or pain not characterized in patients without risk of DVT, even with the use of anticoagulant. It has an acute symptomatology, but may present with chronic manifestations of venous hypertension. A study by Nazzal in 2014 stated that a stenosis with a diameter greater than $70 \%$ was present in $30.6 \%$ of the cases, with a higher incidence among multiparous women [8]. In view of this, the patient in question being multiparous, thrombophilia and carrying the MTS has the corresponding risk factors for this complication, making early correction both surgical and anticoagulant with the best option for its prevention. After the varicose vein surgery, the patient presented a marked improvement in symptoms, but with a persistent sensation of weight in the lower limbs, which progressed and culminated in a later confirmation of Pelvic Congestion Syndrome. The chosen procedure was the embolization of these varicose veins, which proved to be very effective considering that shortly after the patient was asymptomatic. At the moment, she only has pain that is not well-defined in the lower belly. The endovascular treatment of the May-Thurner and Pelvic Congestion Syndrome is safe and has excellent primary patency in the medium to long term (our case 8 years of angioplasty with stent - without intercurrences / 3 years of embolization) as demonstrated by Titus et al in 2011 [9] and Park in 2014 [10].

\section{Conclusion}

The endovascular treatment of the May-Thurner and Pelvic Congestion Syndrome is safe and has excellent primary patency in the medium to long term.

This article demonstrates the importance of giving attention to patient complaints and keeping the research to them.

In addition, we must investigate and treat the diseases before their onset complications.

\section{Authors' Contribution}

Diego Victor Nascimento - bibliographical and written surveys

Gabrielle Maria de Oliveira Kraychete da Silveira bibliographical and written surveys

Helena Chaves de Queiroga - bibliographical and written surveys

Lidie Anne Diniz Viégas - bibliographical and written surveys

Sérgio Ricardo Ferreira Vieira - anesthetist in the surgeries

Francisco Chavier Vieira Bandeira - 1st assistant in bypass surgery

Paulo Roberto da Silva Lima - bibliographic survey, organization and writing of article, besides being the main surgeon in the surgeries

\section{Appendix}

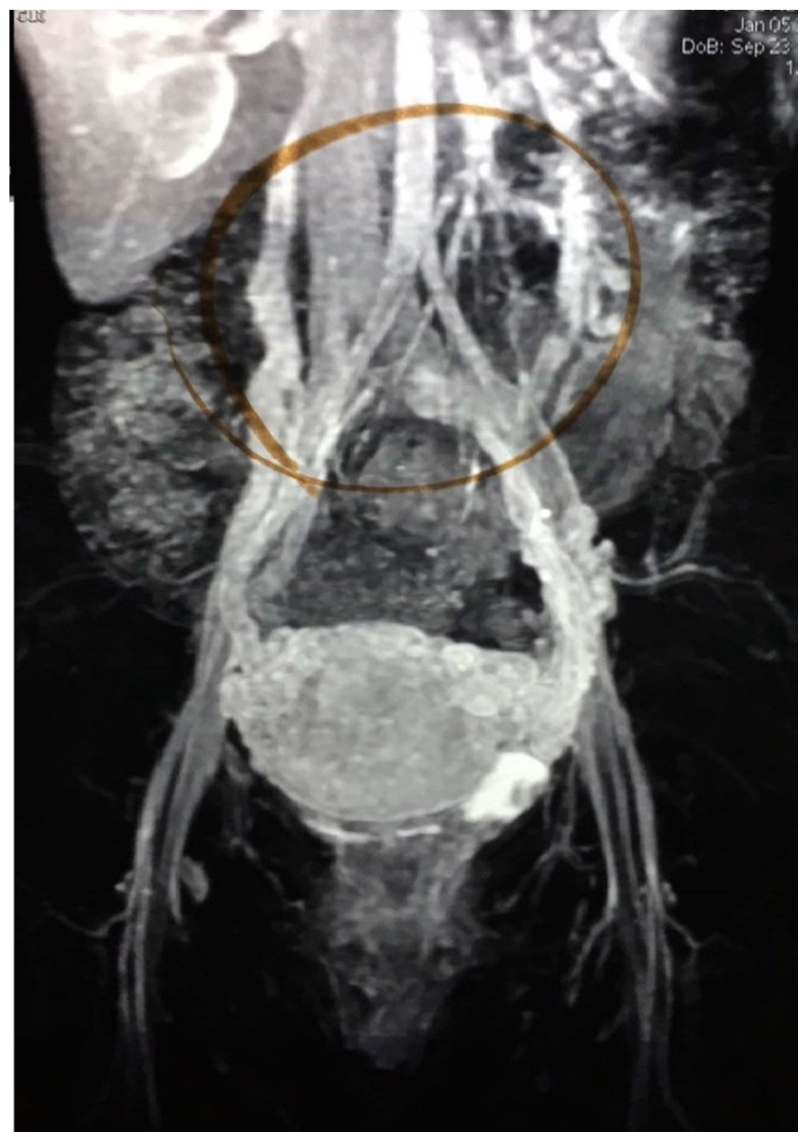

Figure 1. Angio-resonance of the iliac vessels (05/01/2009), evidencing the compression area (gold circle) of Left Common Iliac Vein by Right Common Iliac Artery. In addition to varicose veins around the uterus and Ovarian Veins dilated and communicated with this varicose vein web. 


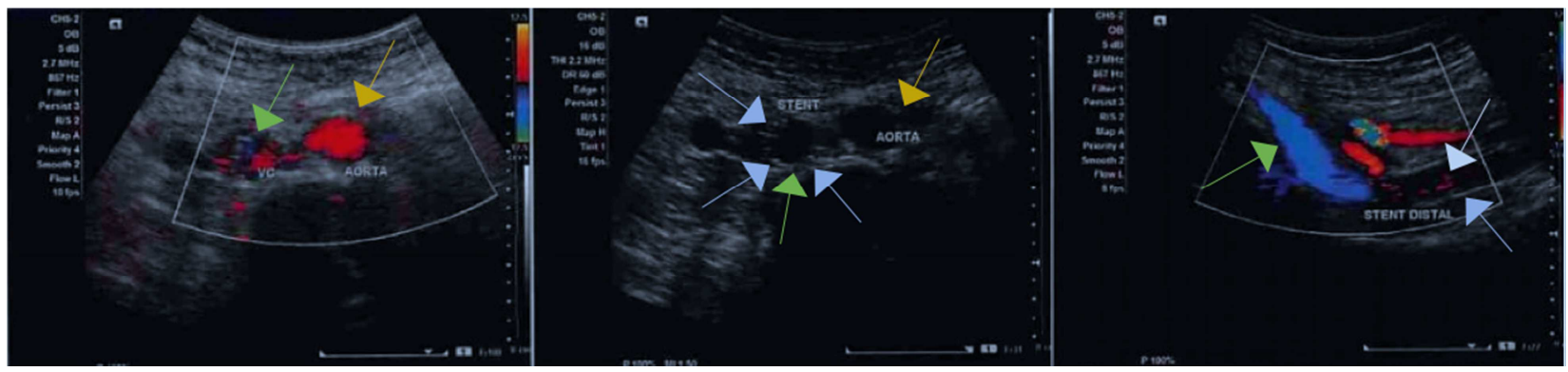

Figure 2. In the left figure, the relationship between into Inferior Vena Cava (green arrow) and the aortic artery (orange arrow) at Doppler effect. In the middle figure, the relationship between the stent (blue arrow) into the Inferior Vena Cava (green arrow) and the aortic artery (orange arrow). In the right figure: Doppler echography of the venous iliac showing patent stent (blue arrow) in Left Common Iliac Vein (white arrow) and Inferior Vena Cava (green arrow).

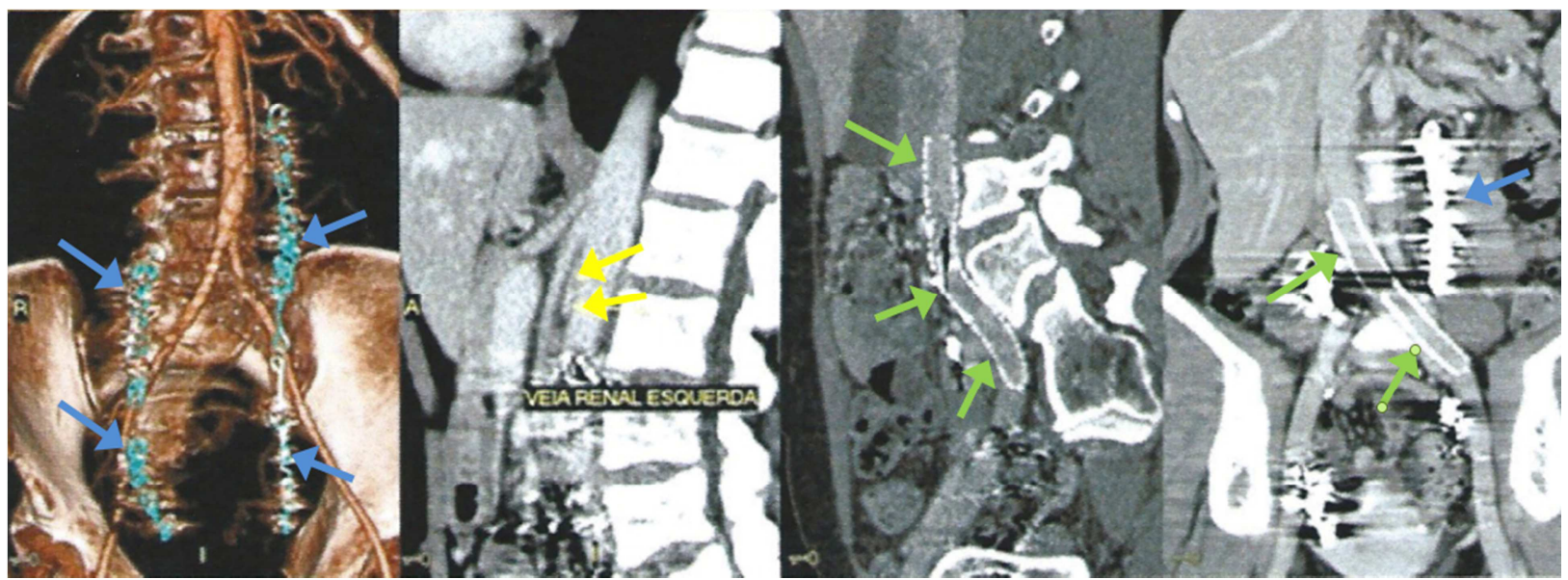

Figure 3. Venous angiotomography of the abdomen and pelvis (01/23/2015): in blue color the embolization (blue arrows) in of the gonadal veins (First-Left figure and last-right figure). Nutcracket Syndrome (Second-Left figure / yellow arrows). Iliocaval stents - Green arrows (First and second-right figures).

\section{References}

[1] Cunha JJRd, Neves DQ, Fontes FA, Solano GP, Cardoso MCT, Lima MHd, et al. Endovascular treatment of the iliac vein compression syndrome (May-Thurner): case report. Jornal Vascular Brasileiro 2011; 10 (1):72-6.

[2] Butros SR, Liu R, Oliveira GR, Ganguli S, Kalva S. Venous compression syndromes: clinical features, imaging findings and management. Br J Radiol. 2013; 86 (1030):20130284.

[3] Cavalcante LP, Souza JEdS, Pereira RM, Bernardes MV, Amanajás AMdS, Parisati MH, et al. Iliac vein compression syndrome: literature review. Jornal Vascular Brasileiro. 2015; $14(1): 78-83$.

[4] Calvaresi E, Swaminathan M, Jokela J. A Case of May-Thurner Syndrome. Carle Sel Pap. 2016; 59 (1):46-47. PMID:27489392.

[5] Galego GdN, Silveira PG, Bortoluzzi CT, Franklin RN, Ronchi TM. Pelvic Congestion Syndrome case series: results of endovascular treatment. Jornal Vascular Brasileiro. 2015; 14 (3):262-6.
[6] Daniels J, Champaneria R, Shah L, Gupta J, Birch J, Moss J. Effectiveness of Embolization or Sclerotherapy of Pelvic Veins for Reducing Chronic Pelvic Pain: A Systematic Review. Journal of vascular and interventional radiology. 2016; 27 (10):1478-86.

[7] Zhang X, Shi X, Gao P, Wang J, Li S, Yao S, et al. Endovascular Management of May-Thurner Syndrome: A Case Report. Medicine (Baltimore). 2016; 95 (4):e2541.

[8] Nazzal M, El-Fedaly M, Kazan V, Qu W, Renno AW, Al-Natour M, et al. Incidence and clinical significance of iliac vein compression. Vascular. 2015; 23 (4):337-43.

[9] Titus, Jessica M. et al. Iliofemoral stenting for venous occlusive disease. J Vasc Surg. 2011;Mar; 53 (3):706-12. doi: 10.1016/j.jvs.2010.09.011. Epub 2010 Dec 3. PMID:21129907.

[10] Park JY, Ahn JH, Jeon YS, Cho SG, Kim JY, Hong KC. Iliac vein stenting as a durable option for residual stenosis after catheter-directed thrombolysis and angioplasty of iliofemoral deep vein thrombosis secondary to May-Thurner syndrome. Phlebology. $2014 \quad$ Aug; $29 \quad$ (7):461-70. doi:10.1177/0268355513491724. Epub 2013 May 28. PMID:23761876. 